\title{
LINEAR OPERATORS ON MATRICES: PRESERVING SPECTRUM AND DISPLACEMENT STRUCTURE
}

\author{
KENNETH R. DRIESSEL* AND WASIN SO ${ }^{\dagger}$
}

\begin{abstract}
In this paper we characterize those linear operators on general matrices that preserve singular values and displacement rank. We also characterize those linear operators on Hermitian matrices that preserve eigenvalues and displacement inertia.
\end{abstract}

Key words. linear operator, displacement structure, Toeplitz

AMS(MOS) subject classification. 15A04

1. Introduction. We introduce some notation to facilitate our discussion.

$$
\begin{aligned}
C^{m \times n} & :=\text { the set of all } m \times n \text { complex matrices; } \\
G l(m) & :=\text { the set of all nonsingular } m \times m \text { matrices; } \\
\operatorname{Herm}(m) & :=\text { the set of all } m \times m \text { Hermitian matrices; } \\
U(m) & :=\text { the set of all } m \times m \text { unitary matrices. }
\end{aligned}
$$

For $1 \leq i \leq m, 1 \leq j \leq n$, let $E^{i j}$ denote the $m \times n$ matrix with 0 everywhere except 1 at the $(i, j)$ position. Then $\left\{E^{i j}\right\}$ is a basis for $C^{m \times n}$. We also adopt the following notation.

$$
\begin{aligned}
\operatorname{sing}(A) & :=\text { the singular values of a matrix } A \text { (including multiplicity); } \\
\text { eigen }(A) & :=\text { the eigenvalues of a Hermitian matrix } A \text { (including multiplicity); } \\
\operatorname{rank}(A) & :=\text { the rank of a matrix } A ; \\
\text { inertia }(A) & :=\text { the inertia of a Hermitian matrix } A .
\end{aligned}
$$

For $A \in C^{m \times n}, \operatorname{ran} k(A)=k$ if and only if $A$ has exactly $k$ nonzero singular values. For $A \in H \operatorname{Herm}(m)$, inertia $(A)=(p, n, z)$ if and only if $A$ has $p$ positive, $n$ negative and $z$ zero eigenvalues, $m=p+n+z$.

We are interested in the spectral properties of matrices that are Toeplitz or nearly Toeplitz. As a consequence, we are interested in linear operators that preserve these properties. We know of only one previous result in this direction. It is the following theorem due to Chu [1992]. Let $E_{m}$ denote the $m \times m$ exchange matrix defined by

$$
E_{m}(i, j):=\delta(i, m+1-j)
$$

where $\delta$ denotes the Kronecker delta. For example, when $m=3$,

$$
E_{3}=\left(\begin{array}{lll}
0 & 0 & 1 \\
0 & 1 & 0 \\
1 & 0 & 0
\end{array}\right)
$$

We write $E$ in place of $E_{m}$ when $m$ is easily determined from the context.

\footnotetext{
* Institute for Mathematics and its Applications, University of Minnesota, Minneapolis, MN 55455 and Department of Mathematics, Idaho State University, Pocatello, ID 83209-8085. driessel@ima .umn.edu

$\dagger$ Institute for Mathematics and its Applications, University of Minnesota, Minneapolis, MN 55455. so@ima . umn . edu
} 
THEOREM 1.1. Let $Q$ be an $m \times m$ orthogonal matrix. Then the following conditions are equivalent:

$\left.{ }^{*}\right)$ If $A$ is an $m \times m$ symmetric Toeplitz matrix then so is $Q A Q^{T}$.

(**) The matrix $Q$ is one of the following:

$$
\pm I, \pm E, \pm I^{\prime}, \pm I^{\prime} E
$$

where $I$ denotes the $m \times m$ identity matrix and $I^{\prime}:=\operatorname{Diag}\left(-1,(-1)^{2}, \ldots,(-1)^{m-1}\right)$.

His techniques can be used to characterize nonzero linear operators on Hermitian matrices which preservse both eigenvalues and Toeplitz structure. We shall report results along this line in a future paper.

In this report, we study the nonzero linear operators which preserve spectra and displacement structure. We begin by recalling the relevant definitions. Let $Z_{m}$ denote the $m \times m$ (lower) shift matrix defined by

$$
Z_{m}(i, j):=\delta(i, j+1)
$$

For example, when $m=3$,

$$
Z_{3}=\left(\begin{array}{lll}
0 & 0 & 0 \\
1 & 0 & 0 \\
0 & 1 & 0
\end{array}\right)
$$

We write $Z$ in place of $Z_{m}$ when $m$ is easily determined from the context. Let $\nabla$ be the linear operator defined by

$$
\nabla:=C^{m \times n} \longrightarrow C^{m \times n}: X \longrightarrow X-Z_{m} X Z_{n}^{T}
$$

For $A \in C^{m \times n}$, the displacement rank of $A$ is defined as

$$
\operatorname{dis}-\operatorname{rank}(A):=\operatorname{rank}(\nabla \cdot A)
$$

In the case $m=n, \nabla$ preserves Hermitian matrices. For $A \in H \operatorname{Her}(m)$, the displacement inertia of $A$ is defined as

$$
\text { dis-inertia }(A):=\text { inertia }(\nabla \cdot A) \text {. }
$$

Kailath appears to be one of the first to emphasize the importance of the displacement structure of matrices. We recall a few of the major results in this area in order to illustrate the significance of these concepts. Note that Toeplitz matrices usually have displacement rank 2. Hence matrices with low displacement rank are regarded as being "nearly Toeplitz". The following result shows that displacement rank is preserved (loosely speaking) under inversion. It is from Kailath, Kung and Morf [1979].

THEOREM 1.2. For $A \in G l(m)$, dis-rank $\left(A^{-1}\right)=\operatorname{dis}-\operatorname{rank}(E A E)$.

The following inequality, due to Comon [1992], shows that if $A$ has small displacement rank then so does its pseudo-inverse $A^{+}$:

$$
\operatorname{dis-rank}\left(A^{+}\right) \leq 2 \text { dis-rank }(E A E) .
$$

Note that Hermitian Toeplitz matrices usually have displacement inertia $(1,1, m-$ 2). Hence Hermitian matrices with low displacement inertia are regarded as being "nearly Toeplitz". Similar to displacement rank, displacement inertia is preserved 
(loosely speaking) under inversion. We learned about this theorem from Tiberiu Constantinescu (Institute of Mathematics of the Romanian Academy of Sciences).

THEOREM 1.3. For $A \in G l(m) \cap H e r m(m)$, dis-inertia $\left(A^{-1}\right)=\operatorname{dis-inertia}(E A E)$. Other versions of displacement structure can be defined and theorems analogous to the last two can often be proved too. See Chun and Kailath [1991], Heinig and Rost [1984].

The rest of this paper is organized as follows. In section 2, we shall characterize those linear operators on general matrices that preserve both rank and displacement rank. As a consequence, we obtain the characterization of those linear operators preserving singular values and displacement rank. The aim of section 3 is to characterize those linear operators on Hermitian matrices that preserve inertia and displacement inertia. We also obtain the characterization of those linear operators preserving eigenvalues and displacement inertia. Then we have some concluding remarks in the final section.

Acknowledgment. This research was done during the 1991-1992 academic year which the authors spent at the Institute for Mathematics and its Applications (IMA) at the University of Minnesota. We thank the members of the IMA for their hospitality. We thank Jack Conn (Department of Mathematics, University of Minnesota) for his suggestions which streamlined some of the proofs. We also thank Leiba Rodman (Department of Mathematics, College of William and Mary) for his comments.

2. Preserving rank and displacement rank. In this section, we shall characterize those nonzero linear operators on $C^{m \times n}$ that preserve both rank and displacement rank. We shall also characterize those that preserve singular values and displacement rank. Recall that two matrices $A, B \in C^{m \times n}$ are equivalent if there exist $M \in G l(m), N \in G l(n)$ such that $B=M A N$. Note that $A$ and $B$ are equivalent if and only if $\operatorname{rank}(A)=\operatorname{rank}(B)$. The following theorem appears in Horn, Li and Tsing [1991]. It characterizes the linear operators preserving equivalence.

THEOREM 2.1. Let $T: C^{m \times n} \longrightarrow C^{m \times n}$ be a nonzero linear operator. Then the following conditions are equivalent:

$\left.{ }^{*}\right)$ T.A is equivalent to T.B whenever $A$ is equivalent to $B$.

$\left(^{* *}\right)$ There exist $M \in G l(m), N \in G l(n)$ such that either, for all $X \in C^{m \times n}, T . X=$ $M X N$ or $m=n$ and, for all $X \in C^{m \times n}, T . X=M X^{T} N$.

As consequences of this result, we obtain the characterization of linear operators preserving rank, and those preserving singular values.

THEOREM 2.2. Let $T: C^{m \times n} \longrightarrow C^{m \times n}$ be a nonzero linear operator. Then the following conditions are equivalent:

$\left(^{*}\right)$ For all $X \in C^{m \times n}, \operatorname{rank}(T . X)=\operatorname{rank}(X)$.

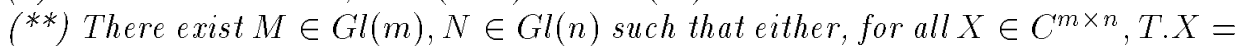
$M X N$ or $m=n$ and, for all $X \in C^{m \times n}, T . X=M X^{T} N$.

Proof. $(* *) \Rightarrow(*)$. Direct verification. $(*) \Rightarrow(* *)$. If $T$ preserves rank then it also preserves equivalence. Hence $T$ has the required forms by Theorem 2.1. $\square$

Theorem 2.3. Let $T: C^{m \times n} \longrightarrow C^{m \times n}$ be a nonzero linear operator. Then the following conditions are equivalent:

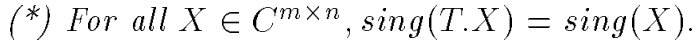

(**) There exist $U \in U(m), V \in U(n)$ such that either, for all $X \in C^{m \times n}, T . X=$ $U X V$ or $m=n$ and, for all $X \in C^{m \times n}, T . X=U X^{T} V$.

Proof. $(* *) \Rightarrow(*)$. Direct verification. $(*) \Rightarrow(* *)$. If $T$ preserves singular values then it also preserves rank. By Theorem 2.2, there exist $M \in G l(m), N \in G l(n)$ 
such that either T.X $=M X N$ or $m=n$ and $T . X=M X^{T} N$. By the singular value decomposition, $M=U_{1} \Sigma_{1} U_{2}$ and $N=V_{1} \Sigma_{2} V_{2}$ where $U_{i} \in U(m), V_{i} \in U(n), \Sigma_{1}=$ $\operatorname{Diag}\left(a_{1}, \ldots, a_{m}\right), \Sigma_{2}=\operatorname{Diag}\left(b_{1}, \ldots, b_{n}\right)$. We consider the case when T.X $=$ MXN. If $X=U_{2}^{*} E^{i j} V_{1}^{*}$ then T.X $=U_{1} \Sigma_{1} E^{i j} \Sigma_{2} V_{2}$. Since $\operatorname{sing}(X)=\operatorname{sing}(T . X)$, we have $a_{i} b_{j}=1$. Consequently, $a_{1}=\cdots=a_{m}=: a, b_{1}=\cdots=b_{n}=: b$ and $a b=1$. This implies that T.X $=U X V$ where $U:=U_{1} U_{2}$ and $V:=V_{1} V_{2}$. The proof is similar for the other case.

Now we characterize those linear operators on $C^{m \times n}$ preserving both rank and displacement rank. For $\lambda \in C$, we shall use $D_{n}(\lambda)$ to denote the $n \times n$ diagonal matrix with diagonal entries $1, \lambda, \ldots, \lambda^{n-1}$; in symbols

$$
D_{n}(\lambda):=\operatorname{Diag}\left(1, \lambda, \ldots, \lambda^{n-1}\right) .
$$

Theorem 2.4. Let $T: C^{m \times n} \longrightarrow C^{m \times n}$ be a nonzero linear operator. Then the following conditions are equivalent:

$\left(^{*}\right)$ For all $X \in C^{m \times n}, \operatorname{rank}(T . X)=\operatorname{rank}(X)$ and dis-rank $(T . X)=\operatorname{dis-rank}(X)$.

(**) There exist $\lambda \neq 0$ and lower triangular Toeplitz matrices $M \in G l(m), N \in$ $G l(n)$ such that either, for all $X \in C^{m \times n}, T . X=D_{m}(\lambda) M X N^{T} D_{n}\left(\lambda^{-1}\right)$ or $m=n$ and, for all $X \in C^{m \times n}, T . X=D_{m}(\lambda) M X^{T} N^{T} D_{n}\left(\lambda^{-1}\right)$.

Before we prove this theorem, we need some preliminary lemmas. The first one is a characterization of matrices that nearly commute with the shift matrix.

Lemma 2.5. Let $B \in C^{n \times n}$ and $\lambda \neq 0$. Then the following conditions are equivalent:

$\left(^{*}\right) B Z_{n}=\lambda Z_{n} B$.

$\left.{ }^{* *}\right)$ There exists a lower triangular Toeplitz matrix $L$ such that $B=D_{n}(\lambda) L$.

Proof. First we observe that $D_{n}(\lambda) Z_{n}=\lambda Z_{n} D_{n}(\lambda)$. (*) $\Rightarrow(* *)$. Let $L:=$ $D_{n}\left(\lambda^{-1}\right) B$. Then $L Z_{n}=Z_{n} L$. By comparing entries, one deduces that $L$ is a lower triangular Toeplitz matrix. $(* *) \Rightarrow(*)$. Since $L$ is a lower triangular Toeplitz matrix, there exists a polynomial $p(x)$ such that $L=p\left(Z_{n}\right)$. Hence $B Z_{n}=D_{n}(\lambda) L Z_{n}=$ $D_{n}(\lambda) p\left(Z_{n}\right) Z_{n}=D_{n}(\lambda) Z_{n} p\left(Z_{n}\right)=\lambda Z_{n} D_{n}(\lambda) p\left(Z_{n}\right)=\lambda Z_{n} D_{n}(\lambda) L=\lambda Z_{n} B$. प

Next we collect some basic results about the Kronecker product. For $A \in C^{n \times n}$ and $B \in C^{m \times m}$, recall that $A \otimes B: C^{m \times n} \longrightarrow C^{m \times n}$ is a linear operator which may be defined by

$$
(A \otimes B) . X:=B X A^{T} .
$$

We prefer this "coordinate-free" definition to the usual one. (Compare Horn and Johnson [1991] or Graham [1981] or Lancaster and Tismenetsky [1985] ). A fundamental property (which is easy to verify using this definition) is that

$$
(A \otimes B) \circ(C \otimes D)=(A C \otimes B D)
$$

where 0 denotes the composition of two operators. Moreover it can be proved that eigen $(A \otimes B)=\left\{\alpha_{i} \beta_{j}: 1 \leq i \leq n, 1 \leq j \leq m\right\}$ where eigen $(A)=\left\{\alpha_{i}: 1 \leq i \leq n\right\}$ and eigen $(B)=\left\{\beta_{j}: 1 \leq j \leq m\right\}$. Hence $\operatorname{tr}(A \otimes B)=(\operatorname{tr} A)(\operatorname{tr} B)$. The next result, which is taken from Marcus and Moyls [1959], is a form of uniqueness for Kronecker product representations.

Lemma 2.6. Let $X_{i}, W_{i} \in C^{n \times n}$ and $Y_{i}, V_{i} \in C^{m \times m}$. If $\sum_{i=1}^{r} X_{i} \otimes Y_{i}=$ $\sum_{i=1}^{s} W_{i} \otimes V_{i}$. and the $X_{i}$ are linearly independent then each $Y_{i} \in \operatorname{Span}\left\{V_{1}, \ldots, V_{s}\right\}$. 
Proof. Since the $X_{j}$ are linearly independent, for each $i$ there exists $P_{i}$ such that $\operatorname{tr}\left(P_{i} X_{j}\right)=\delta(i, j)$. Then, by "contraction", we have

$$
Y_{i}=\sum_{j=1}^{s} \operatorname{tr}\left(P_{i} W_{j}\right) V_{j} .
$$

Corollary 2.7. Let $X_{i} \in C^{n \times n}$ and $Y_{j} \in C^{m \times m}$. If $\left\{X_{i}\right\}$ and $\left\{Y_{j}\right\}$ are linearly independent sets of matrices then $\left\{X_{i} \otimes Y_{j}\right\}$ is a linearly independent set.

Proof. Assume $\sum_{i, j} a_{i j} X_{i} \otimes Y_{j}=0$. We rewrite this equation as

$$
\sum_{i}\left[X_{i} \otimes\left(\sum_{j} a_{i j} Y_{j}\right)\right]=0 .
$$

Use Lemma 2.6 (with all $W_{i}=0$ ) to conclude that, for all $i$,

$$
\sum_{j} a_{i j} Y_{j}=0
$$

Since the $Y_{j}$ are linearly independent, $a_{i j}=0$ for all $i, j$.

Corollary 2.8. Let $X \in G l(n), Y \in G l(m)$. Then $\left\{X \otimes Y, Z_{n} X \otimes Y, X \otimes\right.$ $\left.Z_{m} Y, Z_{n} X \otimes Z_{m} Y\right\}$ is a linearly independent set.

Proof. Since $X \in G l(n),\left\{X, Z_{n} X\right\}$ is a linearly independent set. Similarly $\left\{Y, Z_{m} Y\right\}$ is a linearly independent set. Apply Corollary 2.7 to obtain the required result. $\square$

The following result appears in Horn and Johnson [1991] and Graham [1981].

Lemma 2.9. Let trans $:=C^{n \times n} \longrightarrow C^{n \times n}: X \longrightarrow X^{T}$ denote the linear operator of taking transpose. Then trans has the following Kronecker product representation:

$$
\text { trans }=\sum_{i, j=1}^{n} E^{i j} \otimes E^{j i}
$$

where $E^{i j}$ is the $n \times n$ matrix with 0 everywhere except 1 at the $(i, j)$ position.

Proof. For $X=\left(x_{i j}\right) \in C^{n \times n}$, note that $E^{j i} X E^{j i}=x_{i j} E^{j i}$. Now we have

$$
\text { trans. } X=X^{T}=\sum_{i, j} x_{i j} E^{j i}=\sum_{i, j} E^{j i} X E^{j i}=\sum_{i, j} E^{i j} \otimes E^{j i} \cdot X .
$$

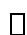

We adopt the convention that $E^{i j}=0$ if $i>n, j>n, i<1$, or $j<1$. Then it is easy to verify that $Z E^{i j}=E^{(i+1) j}$, and $E^{i j} Z=E^{i(j-1)}$. With this observation, we are ready to prove the next lemma.

Lemma 2.10. If $P, Q, R, S \in C^{n \times n}$ are such that

$$
\left(I_{n} \otimes I_{n}-Z_{n} \otimes Z_{n}\right) \circ(Q \otimes P)=(S \otimes R) \circ \text { trans } \circ\left(I_{n} \otimes I_{n}-Z_{n} \otimes Z_{n}\right)
$$

then at least one of $\{P, R, S\}$ is singular.

Proof. Assume that $P, R, S \in G l(n)$. By Lemma 2.9, trans $=\sum_{i, j=1}^{n} E^{i j} \otimes E^{j i}$. Hence

$$
\left(I_{n} \otimes I_{n}-Z_{n} \otimes Z_{n}\right)(Q \otimes P)=(S \otimes R)\left(\sum_{i, j=1}^{n} E^{i j} \otimes E^{j i}\right)\left(I_{n} \otimes I_{n}-Z_{n} \otimes Z_{n}\right) .
$$


Since

$$
\begin{aligned}
\sum_{i, j=1}^{n}\left(E^{i j} \otimes E^{j i}\right)\left(I_{n} \otimes I_{n}-Z_{n} \otimes Z_{n}\right) & =\sum_{i, j=1}^{n} E^{i j} \otimes E^{j i}-E^{i(j-1)} \otimes E^{j(i-1)} \\
& =\sum_{i, j=1}^{n} E^{i j} \otimes E^{j i}-E^{i j} \otimes E^{(j+1)(i-1)} \\
& =\sum_{i, j=1}^{n} E^{i j} \otimes\left(E^{j i}-E^{(j+1)(i-1)}\right)
\end{aligned}
$$

we have

$$
Q \otimes P-Z_{n} Q \otimes Z_{n} P=\sum_{i, j=1}^{n} S E^{i j} \otimes R\left(E^{j i}-E^{(j+1)(i-1)}\right)
$$

Note that $\left\{S E^{i j}\right\}$ is a linearly independent set. Use Lemma 2.6 to conclude that, for all $i, j, R\left(E^{j i}-E^{(j+1)(i-1)}\right) \in \operatorname{Span}\left\{P, Z_{n} P\right\}$. In particular, $R E^{11}, R E^{21}, R\left(E^{12}-E^{21}\right) \in$ $\operatorname{Span}\left\{P, Z_{n} P\right\}$. Hence

$$
3=\operatorname{dim} \operatorname{Span}\left\{R E^{11}, R E^{21}, R\left(E^{12}-E^{21}\right)\right\} \leq \operatorname{dim} \operatorname{Span}\left\{P, Z_{n} P\right\}=2 .
$$

This is a contraction. $\square$

LEMMA 2.11. If $P, R \in G l(m)$ and $Q, S \in G l(n)$ satisfy

$$
\left(I_{n} \otimes I_{m}-Z_{n} \otimes Z_{m}\right) \circ(Q \otimes P)=(S \otimes R) \circ\left(I_{n} \otimes I_{m}-Z_{n} \otimes Z_{m}\right)
$$

then there exist $\lambda \neq 0$ and lower triangular Toeplitz matrices $N \in G l(n), M \in G l(m)$ such that $Q=D_{n}\left(\lambda^{-1}\right) N$ and $P=D_{m}(\lambda) M$.

Proof. Note that we can rewrite the given equation as follows:

$$
Q \otimes P-Z_{n} Q \otimes Z_{m} P=S \otimes R-S Z_{n} \otimes R Z_{m}
$$

By Lemma 2.6, $S, S Z_{n} \in \operatorname{Span}\left\{Q, Z_{n} Q\right\}$, i.e. there exist $\alpha, \beta, \gamma, \delta \in C$ such that

$$
S=\alpha Q+\beta Z_{n} Q \text { and } S Z_{n}=\gamma Q+\delta Z_{n} Q
$$

Note that $S=\left(\alpha I+\beta Z_{n}\right) Q$ has rank $n$; hence $\alpha \neq 0$. Also note that $S Z_{n}=$ $\left(\gamma I+\delta Z_{n}\right) Q$ has rank $n-1$; hence $\gamma=0$. Furthermore $0 \neq S Z_{n}=\delta Z_{n} Q$ and hence $\delta \neq 0$. In summary, we have

$$
S=\alpha Q+\beta Z_{n} Q \text { and } S Z_{n}=\delta Z_{n} Q
$$

where $\alpha \neq 0$ and $\delta \neq 0$. Similarly, we get

$$
R=a P+b Z_{m} P \text { and } R Z_{m}=d Z_{m} P
$$

where $a \neq 0$ and $d \neq 0$. Substituting back into the equation (1), we deduce that, by Corollary 2.8, $\beta=0, b=0$ and $\alpha a=\delta d=1$. Thus

$$
\begin{aligned}
& S=\alpha Q, S Z_{n}=\delta Z_{n} Q \\
& R=a P, R Z_{m}=d Z_{m} P
\end{aligned}
$$


and hence

$$
\lambda^{-1} Z_{n} Q=Q Z_{n} \quad \text { and } \quad \lambda Z_{m} P=P Z_{m}
$$

where $\lambda:=\frac{\alpha}{\delta}=\frac{d}{a}$. By Lemma 2.5,

$$
Q=D_{n}\left(\lambda^{-1}\right) N \quad \text { and } \quad P=D_{m}(\lambda) M
$$

where $M, N$ are lower triangular Toeplitz matrices of dimension $m, n$ respectively.

We are now ready to prove Theorem 2.4 .

Proof. $(* *) \Rightarrow(*)$. It is clear that $T$ preserves rank. It remains to show that $T$ preserves displacement rank.

Case 1. T.X $=D_{m}(\lambda) M X N^{T} D_{n}\left(\lambda^{-1}\right)$

By Lemma 2.5, we have

$$
\begin{aligned}
T . X-Z_{m}(T . X) Z_{n}^{T} & =D_{m}(\lambda) M X N^{T} D_{n}\left(\lambda^{-1}\right)-Z_{m} D_{m}(\lambda) M X N^{T} D\left(\lambda^{-1}\right) Z_{n}^{T} \\
& =D_{m}(\lambda) M X N^{T} D_{n}\left(\lambda^{-1}\right)-\lambda^{-1} D_{m}(\lambda) M Z_{m} X \lambda Z_{n}^{T} N^{T} D\left(\lambda^{-1}\right) \\
& =D_{m}(\lambda) M X N^{T} D_{n}\left(\lambda^{-1}\right)-D_{m}(\lambda) M Z_{m} X Z_{n}^{T} N^{T} D\left(\lambda^{-1}\right) \\
& =D_{m}(\lambda) M\left(X-Z_{m} X Z_{n}^{T}\right) N^{T} D\left(\lambda^{-1}\right) .
\end{aligned}
$$

Hence

$$
\begin{aligned}
\operatorname{dis} \operatorname{rank}(T . X) & =\operatorname{rank}\left(T . X-Z_{m}(T . X) Z_{n}^{T}\right) \\
& =\operatorname{rank}\left(X-Z_{m} X Z_{n}^{T}\right) \\
& =\operatorname{dis} \operatorname{rank}(X) .
\end{aligned}
$$

Case 2. T.X $=D_{m}(\lambda) M X^{T} N^{T} D_{n}\left(\lambda^{-1}\right)$

Using an argument like Case 1, we conclude that $T$ preserves displacement rank.

$(*) \Rightarrow(* *)$. We assume that $T$ is a nonzero linear operator that preserves rank and displacement rank. We define $\hat{T}: C^{m \times n} \longrightarrow C^{m \times n}$ by

$$
\hat{T}:=\left(I_{n} \otimes I_{m}-Z_{n} \otimes Z_{m}^{T}\right) \circ T \circ\left(I_{n} \otimes I_{m}-Z_{n} \otimes Z_{m}^{T}\right)^{-1} .
$$

Hence

$$
\left(I_{n} \otimes I_{m}-Z_{n} \otimes Z_{m}^{T}\right) \circ T=\hat{T} \circ\left(I_{n} \otimes I_{m}-Z_{n} \otimes Z_{m}^{T}\right)
$$

Since $T$ preserves rank, by Theorem 2.2, there exist $P \in G l(m), Q \in G l(n)$ such that either $T=Q \otimes P$ or $m=n$ and $T=(Q \otimes P) \circ$ trans. On the other hand, since $T$ preserves displacement rank, it follows that $\hat{T}$ preserve rank. Then, by Theorem 2.2 , there exist $R \in G l(m), S \in G l(n)$ such that either $\hat{T}=S \otimes R$ or $m=n$ and $\hat{T}=(S \otimes R) \circ$ trans. We have 4 cases to consider.

Case 1. $T=Q \otimes P$ and $\hat{T}=S \otimes R$

Note that $\left(I_{n} \otimes I_{m}-Z_{n} \otimes Z_{m}^{T}\right) \circ(Q \otimes P)=(S \otimes R) \circ\left(I_{n} \otimes I_{m}-Z_{n} \otimes Z_{m}^{T}\right)$. Then, by Lemma 2.11, there exist $\lambda \neq 0$ and lower triangular Toeplitz matrices $N \in G l(n), M \in G l(m)$ such that $Q=D_{n}\left(\lambda^{-1}\right) N$ and $P=D_{m}(\lambda) M$. Consequently, $T=D_{n}\left(\lambda^{-1}\right) N \otimes D_{m}(\lambda) M$.

Case 2. $m=n, T=Q \otimes P$ and $\hat{T}=(S \otimes R) \circ$ trans

Note that $\left(I_{n} \otimes I_{m}-Z_{n} \otimes Z_{m}^{T}\right) \circ(Q \otimes P)=(S \otimes R) \circ$ trans $\circ\left(I_{n} \otimes I_{m}-Z_{n} \otimes Z_{m}^{T}\right)$.

Then, by Lemma 2.10, one of $\{P, R, S\}$ is singular, a contradiction. 
Case 3. $m=n, T=(Q \otimes P) \circ$ trans and $\hat{T}=S \otimes R$

Note that $\left(I_{n} \otimes I_{m}-Z_{n} \otimes Z_{m}^{T}\right) \circ(Q \otimes P) \circ$ trans $=(S \otimes R) \circ\left(I_{n} \otimes I_{m}-Z_{n} \otimes Z_{m}^{T}\right)$. Using the fact that

$$
\left(I_{n} \otimes I_{m}-Z_{n} \otimes Z_{m}^{T}\right) \circ \text { trans }=\text { trans } \circ\left(I_{n} \otimes I_{m}-Z_{n} \otimes Z_{m}^{T}\right),
$$

we deduce that

$$
\left(I_{n} \otimes I_{m}-Z_{n} \otimes Z_{m}^{T}\right) \circ(Q \otimes P)=(S \otimes R) \circ \text { trans } \circ\left(I_{n} \otimes I_{m}-Z_{n} \otimes Z_{m}^{T}\right) .
$$

Then, by Lemma 2.10, one of $\{P, R, S\}$ is singular, a contradiction.

Case 4. $m=n, T=(Q \otimes P) \circ$ trans and $\hat{T}=(S \otimes R) \circ$ trans

Note that $\left(I_{n} \otimes I_{m}-Z_{n} \otimes Z_{m}^{T}\right) \circ(Q \otimes P) \circ$ trans $=(S \otimes R) \circ$ trans $\circ\left(I_{n} \otimes\right.$ $\left.I_{m}-Z_{n} \otimes Z_{m}^{T}\right)$. Using the fact that

$$
\left(I_{n} \otimes I_{m}-Z_{n} \otimes Z_{m}^{T}\right) \circ \text { trans }=\text { trans } \circ\left(I_{n} \otimes I_{m}-Z_{n} \otimes Z_{m}^{T}\right),
$$

we deduce that

$$
\left(I_{n} \otimes I_{m}-Z_{n} \otimes Z_{m}^{T}\right) \circ(Q \otimes P)=(S \otimes R) \circ\left(I_{n} \otimes I_{m}-Z_{n} \otimes Z_{m}^{T}\right) .
$$

Then, by Lemma 2.11, there exist $\lambda \neq 0$ and lower triangular Toeplitz matrices $N \in G l(n), M \in G l(m)$ such that $Q=D_{n}\left(\lambda^{-1}\right) N$ and $P=D_{m}(\lambda) M$. Consequently, $T=D_{n}\left(\lambda^{-1}\right) N \otimes D_{m}(\lambda) M$.

$\square$

Next we give the characterization of those linear operators on $C^{n \times n}$ preserving both singular values and displacement rank.

THEOREM 2.12. Let $T: C^{m \times n} \longrightarrow C^{m \times n}$ be a nonzero linear operator. Then the following conditions are equivalent:

$\left(^{*}\right)$ For all $X \in C^{m \times n}, \operatorname{sing}(T . X)=\operatorname{sing}(X)$ and dis-rank $(T . X)=\operatorname{dis-rank}(X)$.

$\left.{ }^{* *}\right)$ There exist $|\lambda|=|\mu|=1$ such that either, for all $X \in C^{m \times n}, T . X=$ $\mu D_{m}(\lambda) X D_{n}\left(\lambda^{-1}\right)$ or $m=n$ and, for all $X \in C^{m \times n}, T . X=\mu D_{m}(\lambda) X^{T} D_{n}\left(\lambda^{-1}\right)$.

Proof. $(* *) \Rightarrow(*)$. Since $|\lambda|=|\mu|=1, \mu D_{m}(\lambda)$ and $D_{n}\left(\lambda^{-1}\right)$ are unitary. Hence $T$ preserves singular values. By Theorem 2.4, we know $T$ also preserves displacement rank. $(*) \Rightarrow(* *)$. Since $T$ preserves singular values, by Theorem 2.3 , there exist $U \in$ $U(m), V \in U(n)$ such that either $T . X=U X V$ or $m=n$ and $T . X=U X^{T} V$. On the other hand, since $T$ preserves both rank and displacement rank, by Theorem 2.4, there exist $\lambda \neq 0$ and lower triangular Toeplitz matrices $M \in G l(m), N \in G l(n)$ such that either T.X $=D_{m}(\lambda) M X N^{T} D_{n}\left(\lambda^{-1}\right)$ or $m=n$ and T.X $=D_{m}(\lambda) M X^{T} N^{T} D_{n}\left(\lambda^{-1}\right)$. We consider the following 4 cases.

Case 1. T. $X=D_{m}(\lambda) M X N^{T} D_{n}\left(\lambda^{-1}\right)$ and T.X $=U X V$.

For all $X \in C^{m \times n}, D_{m}(\lambda) M X N^{T} D_{n}\left(\lambda^{-1}\right)=U X V$. Then there exists $\alpha \in C$ such that $\alpha D_{m}(\lambda) M=U$ and $\frac{1}{\alpha} N^{T} D_{n}\left(\lambda^{-1}\right)=V$. Therefore both $\alpha D_{m}(\lambda) M$ and $\frac{1}{\alpha} N^{T} D_{n}\left(\lambda^{-1}\right)$ are diagonal and so $M=u I_{m}$ and $N=v I_{n}$ for some $u, v \in$ C. Moreover $|\lambda|=|\alpha|=|u v|=1$. Consequently, T.X $=\mu D_{m}(\lambda) X D_{n}\left(\lambda^{-1}\right)$ where $\mu:=u v$.

Case 2. $m=n, T . X=D_{m}(\lambda) M X N^{T} D_{n}\left(\lambda^{-1}\right)$ and T.X $=U X^{T} V$.

For all $X \in C^{m \times n}, D_{m}(\lambda) M X N^{T} D_{n}\left(\lambda^{-1}\right)=U X^{T} V$. Evaluating at $X=I_{n}$, we get $D_{m}(\lambda) M N^{T} D_{n}\left(\lambda^{-1}\right)=U V$. Let $W:=U^{*} D_{m}(\lambda) M=V D_{n}(\lambda) N^{-T}$. Then $W X=X^{T} W$ for all $X \in C^{m \times n}$. In particular, $W$ commutes with every diagonal matix. Hence $W$ is a scalar, and so $X=X^{T}$ for all $X \in C^{m \times n}$, a contradiction. 
Case 3. $m=n$, T.X $=D_{m}(\lambda) M X^{T} N^{T} D_{n}\left(\lambda^{-1}\right)$ and T.X $=U X V$.

Using the same argument as in Case 2, we conclude that case 3 is impossible.

Case 4. $m=n, T . X=D_{m}(\lambda) M X^{T} N^{T} D_{n}\left(\lambda^{-1}\right)$ and T.X $=U X^{T} V$.

Using the same argument as in Case 1 , we conclude that T.X $=\mu D_{m}(\lambda) X^{T} D_{n}\left(\lambda^{-1}\right)$. $\square$

3. Preserving inertia and displacement inertia. In this section, we shall characterize those nonzero linear operators on $\operatorname{Herm}(n)$ that preserve both inertia and displacement inertia. We shall also characterize those preserving eigenvalues and displacement inertia. Recall that two matrices $A, B \in \operatorname{Herm}(n)$ are *-congruent if there exists $S \in G l(n)$ such that $B=S A S^{*}$. Note that $A$ and $B$ are *-congruent if and only if inertia $(A)=$ inertia $(B)$. The following theorem appears in Horn, Li and Tsing [1991]. It characterizes the linear operators preserving $*$-congruence. For simplicity, we write $I_{n}$ as $I, Z_{n}$ as $Z$ and $D_{n}(\lambda)$ as $D(\lambda)$ in the following.

THEOREM 3.1. Let $T: \operatorname{Herm}(n) \longrightarrow \operatorname{Herm}(n)$ be a nonzero linear operator. Then the following conditions are equivalent:

$\left(^{*}\right)$ T. $A$ is *-congruent to T.B whenever $A$ is *-congruent to $B$.

$\left.{ }^{* *}\right)$ There exists $S \in G l(n)$ such that either, for all $X \in \operatorname{Herm}(n), T . X=$ $\pm S X S^{*}$ or, for all $X \in \operatorname{Herm}(n), T . X= \pm S X^{T} S^{*}$.

As consequences of this result, we obtain the characterization of linear operators preserving inertia and those preserving eigenvalues.

THEOREM 3.2. Let $T: \operatorname{Herm}(n) \longrightarrow \operatorname{Herm}(n)$ be a nonzero linear operator. Then the following conditions are equivalent:

$\left(^{*}\right)$ For all $X \in \operatorname{Herm}(n)$, inertia $(T . X)=\operatorname{inertia}(X)$.

$\left(^{* *}\right)$ There exists $S \in G l(n)$ such that either, for all $X \in \operatorname{Herm}(n), T . X=S X S^{*}$ or, for all $X \in \operatorname{Herm}(n), T . X=S X^{T} S^{*}$.

Proof. $(* *) \Rightarrow(*)$. Direct verification. $(*) \Rightarrow(* *)$. If $T$ preserves inertia then it also preserves $*$-congruence. Hence, by Theorem 3.1, there exists $S \in G l(n)$ such that either T.X $= \pm S X S^{*}$ or T.X $= \pm S X^{T} S^{*}$. However the cases with minus signs are ruled out because $T$ preserves inertia.

THEOREM 3.3. Let $T: \operatorname{Herm}(n) \longrightarrow \operatorname{Herm}(n)$ be a nonzero linear operator. Then the following conditions are equivalent:

$\left(^{*}\right)$ For all $X \in \operatorname{Herm}(n)$, eigen $(T . X)=\operatorname{eigen}(X)$.

$\left.{ }^{* *}\right)$ There exists $U \in U(n)$ such that either, for all $X \in H e r m(n), T . X=U X U^{*}$ or, for all $X \in \operatorname{Herm}(n), T . X=U X^{T} U^{*}$.

Proof. $(* *) \Rightarrow(*)$. Direct verification. $(*) \Rightarrow(* *)$. If $T$ preserves eigenvalues then it also preserves inertia. Hence, by Theorem 3.2, there exists $S \in G l(n)$ such that either $T . X=S X S^{*}$ or $T . X=S X^{T} S^{*}$. Since $T$ preserves eigenvalues, eigen $(I)=$ $\operatorname{eigen}(T . I)=\operatorname{eigen}\left(S S^{*}\right)$. Therefore $S S^{*}=I$ and so $S \in U(n)$.

Now we characterize those linear operators on $\operatorname{Herm}(n)$ preserving both inertia and displacement inertia.

THEOREM 3.4. Let T:Herm $(n) \longrightarrow \operatorname{Herm}(n)$ be a nonzero linear operator. Then the following conditions are equivalent:

$\left(^{*}\right)$ For all $X \in \operatorname{Herm}(n)$,inertia $(T . X)=\operatorname{inertia}(X)$ and dis-inertia $(T . X)=$ dis-inertia $(X)$.

$\left.{ }^{* *}\right)$ There exists $|\lambda|=1$ and a lower triangular Toeplitz $N \in G l(n)$ such that either, for all $X \in \operatorname{Herm}(n), T . X=D(\lambda) N X N^{*} D(\lambda)^{*}$ or, for all $X \in \operatorname{Herm}(n), T . X=$ $D(\lambda) N X^{T} N^{*} D(\lambda)^{*}$.

Proof. $(* *) \Rightarrow(*)$. It is clear that $T$ preserves inertia. It remains to show that $T$ preserves displacement inertia. 
Case 1. T.X $=D(\lambda) N X N^{*} D(\lambda)^{*}$

By Lemma 2.5, we have

$$
\begin{aligned}
T . X-Z(T . X) Z^{T} & =D(\lambda) N X N^{*} D(\lambda)^{*}-Z D(\lambda) N X N^{*} D(\lambda)^{*} Z^{T} \\
& =D(\lambda) N X N^{*} D(\lambda)^{*}-\lambda^{-1} D(\lambda) N Z X\left(\lambda^{*}\right)^{-1} Z^{T} N^{*} D(\lambda)^{*} \\
& =D(\lambda) N X N^{*} D(\lambda)^{*}-D(\lambda) N Z X Z^{T} N^{*} D(\lambda)^{*} \\
& =D(\lambda) N\left(X-Z X Z^{T}\right) N^{*} D(\lambda)^{*}
\end{aligned}
$$

Hence

$$
\begin{aligned}
\operatorname{dis-inertia}(T . X) & =\operatorname{inertia}\left(T . X-Z(T . X) Z^{T}\right) \\
& =\operatorname{inertia}\left(X-Z X Z^{T}\right) \\
& =\text { dis-inertia }(X) .
\end{aligned}
$$

Case 2. T.X $=D(\lambda) N X^{T} N^{*} D(\lambda)^{*}$

Using an argument like Case 1 , we conclude that $T$ preserves displacement inertia.

$(*) \Rightarrow(* *)$. We assume that $T$ is a nonzero linear operator that preserves inertia and displacement inertia. We define $\hat{T}: \operatorname{Herm}(n) \longrightarrow \operatorname{Herm}(n)$ by

$$
\hat{T}:=\left(I \otimes I-Z \otimes Z^{T}\right) \circ T \circ\left(I \otimes I-Z \otimes Z^{T}\right)^{-1} .
$$

Hence

$$
\left(I \otimes I-Z \otimes Z^{T}\right) \circ T=\hat{T} \circ\left(I \otimes I-Z \otimes Z^{T}\right) .
$$

Since $T$ preserves inertia, by Theorem 3.2, there exist $S \in G l(n)$ such that either $T=\bar{S} \otimes S$ or $T=(\bar{S} \otimes S)$ otrans. On the other hand, since $T$ preserves displacement inertia, it follows that $\hat{T}$ preserve inertia. Then, by Theorem 3.2, there exist $R \in G l(n)$ such that either $\hat{T}=\bar{R} \otimes R$ or $\hat{T}=(\bar{R} \otimes R) \circ$ trans. We have 4 cases to consider.

Case 1. $T=\bar{S} \otimes S$ and $\hat{T}=\bar{R} \otimes R$

Note that $\left(I \otimes I-Z \otimes Z^{T}\right) \circ(\bar{S} \otimes S)=(\bar{R} \otimes R) \circ\left(I \otimes I-Z \otimes Z^{T}\right)$. Then, by Lemma 2.11, there exist $\lambda \neq 0$ and lower triangular Toeplitz matrices $N, M \in G l(n)$ such that $\bar{S}=D\left(\lambda^{-1}\right) M$ and $S=D(\lambda) N$. Hence $\bar{M}=D\left(|\lambda|^{2}\right) N$, and so $|\lambda|=1$. Consequently, T.X $=D(\lambda) N X(D(\lambda) N)^{*}$ for all $X \in \operatorname{Herm}(n)$.

Case 2. $T=\bar{S} \otimes S$ and $\hat{T}=(\bar{R} \otimes R) \circ$ trans

Note that $\left(I \otimes I-Z \otimes Z^{T}\right) \circ(\bar{S} \otimes S)=(\bar{R} \otimes R) \circ$ trans $\circ\left(I \otimes I-Z \otimes Z^{T}\right)$. By Lemma 2.10, we get a contradiction.

Case 3. $T=(\bar{S} \otimes S) \circ$ trans and $\hat{T}=\bar{R} \otimes R$

Note that $\left(I \otimes I-Z \otimes Z^{T}\right) \circ(\bar{S} \otimes S) \circ$ trans $=(\bar{R} \otimes R) \circ\left(I \otimes I-Z \otimes Z^{T}\right)$. Using the fact that

$$
\left(I \otimes I-Z \otimes Z^{T}\right) \circ \text { trans }=\text { trans } \circ\left(I \otimes I-Z \otimes Z^{T}\right),
$$

we deduce that

$$
\left(I \otimes I-Z \otimes Z^{T}\right) \circ(\bar{S} \otimes S)=(\bar{R} \otimes R) \circ \text { trans } \circ\left(I \otimes I-Z \otimes Z^{T}\right),
$$

which leads to a contradiction by Lemma 2.10 . 
Case 4. $T=(\bar{S} \otimes S) \circ$ trans and $\hat{T}=(\bar{R} \otimes R) \circ$ trans

Note that $\left(I \otimes I-Z \otimes Z^{T}\right) \circ(\bar{S} \otimes S) \circ$ trans $=(\bar{R} \otimes R) \circ$ trans $\circ\left(I \otimes I-Z \otimes Z^{T}\right)$. Using the fact that

$$
\left(I \otimes I-Z \otimes Z^{T}\right) \circ \text { trans }=\text { trans } \circ\left(I \otimes I-Z \otimes Z^{T}\right),
$$

we deduce that

$$
\left(I \otimes I-Z \otimes Z^{T}\right) \circ(\bar{S} \otimes S)=(\bar{R} \otimes R) \circ\left(I \otimes I-Z \otimes Z^{T}\right) .
$$

Then we obtain the required result as in Case 1.

[

Next we give the characterization of those linear operators on $\operatorname{Herm}(n)$ preserving both eigenvalues and displacement inertia.

THEOREM 3.5. Let $T: \operatorname{Herm}(n) \longrightarrow \operatorname{Herm}(n)$ be a nonzero linear operator. Then the following conditions are equivalent:

$\left.{ }^{*}\right)$ For all $X \in \operatorname{Herm}(n)$,eigen $(T . X)=\operatorname{eigen}(X)$ and dis-inertia $(T . X)=$ dis-inertia $(X)$.

(**) There exists $|\lambda|=1$ such that either, for all $X \in \operatorname{Herm}(n), T . X=D(\lambda) X D(\lambda)^{*}$ or, for all $X \in \operatorname{Herm}(n)$, T.X $=D(\lambda) X^{T} D(\lambda)^{*}$.

Proof. $(* *) \Rightarrow(*)$. Since $|\lambda|=1, D(\lambda)$ is unitary and hence $T$ preserves eigenvalues. From Theorem 3.4, it is clear that $T$ also preserves displacement inertia. $(*) \Rightarrow(* *)$. Since $T$ preserves eigenvalues, from Theorem 3.3, there exists $U \in U(n)$ such that either T.X $=U X U^{*}$ or $T . X=U X^{T} U^{*}$. On the other hand, since $T$ preserves both inertia and displacement inertia, from Theorem 3.4, there exists $|\lambda|=1$ and a lower triangular Toeplitz $N \in G l(n)$ such that either T.X $=D(\lambda) N X N^{*} D(\lambda)^{*}$ or T.X $=D(\lambda) N X^{T} N^{*} D(\lambda)^{*}$. We consider the following 4 cases.

Case 1. T. $X=D(\lambda) N X N^{*} D(\lambda)^{*}$ and T.X $=U X U^{*}$.

Evaluating at $X=I$, we get $D(\lambda) N N^{*} D(\lambda)^{*}=U U^{*}=I$, i.e. $D(\lambda) N$ is unitary. Since $D(\lambda) N$ is lower triangular, it must be diagonal and so is $N$. This implies that $N=\mu I$ because $N$ is Toeplitz. Moreover $|\mu|=1$. Finally $T . X=D(\lambda) X D(\lambda)^{*}$.

Case 2. T.X $=D(\lambda) N X N^{*} D(\lambda)^{*}$ and $T . X=U X^{T} U^{*}$.

For all $X \in \operatorname{Herm}(n), D(\lambda) N X N^{*} D(\lambda)^{*}=U X^{T} U^{*}$. Evaluating at $X=I$, we find that $D(\lambda) N$ is unitary. Let $V:=U^{*} D(\lambda) N$. Then $V X=X^{T} V$ for all $X \in \operatorname{Herm}(n)$. This implies that $V$ must be a scalar, and so $X=X^{T}$ for all $X \in \operatorname{Herm}(n)$, a contradiction.

Case 3. T.X $=D(\lambda) N X^{T} N^{*} D(\lambda)^{*}$ and T.X $=U X U^{*}$.

Using the same argument as in Case 2, we conclude that Case 3 is impossible.

Case 4. T.X $=D(\lambda) N X^{T} N^{*} D(\lambda)^{*}$ and T.X $=U X^{T} U^{*}$.

Using the same argument as in Case 1 , we conclude that T.X $=D(\lambda) X^{T} D(\lambda)^{*}$.

4. Concluding Remarks. There are many papers on rank preserving linear operators and inertia preserving linear operators: Adams [1962], Adams, Lax and Phillips [1965], Atkinson [1983], Atkinson and Lloyd [1980,1981], Baruch and Loewy [1991], Beasley [1970,1981,1983,1988], Botta [1987], Eisenbud and Harris [1988], Flanders [1962], Helton and Rodman [1985], Johnson and Pierce [1985,1986], Laffey and Loewy [1990], Lim [1979], Loewy [1989,1990,1991], Loewy and Pierce [1991], Marcus and Moyls [1959], Meshulam [1985,1989], Moore [1966], Pierce and Rodman [1988], Schneider [1965], Sylvester [1986], Westwick [1967,1987]. (We received this list from 
R. Loewy. We have not looked at all these papers.) Some of them characterize linear preservers of one particular rank class or one particular inertia class (rather than characterizing preservers of all rank or inertia classes as was done in Theorem 2.2 and 3.2). These results probably make it possible to characterize linear preservers of one particular rank-and-displacement-rank class and linear preservers of one particular inertia-and displacement-inertia class. Many of the results in these references treat rank preservers or inertia preservers over the field of real numbers (rather than the field of complex numbers that we used in this report). Some of the references even deal with more general fields of numbers. These preserver results over other fields probably make it possible to extend the results of this report to other fields of numbers.

We mentioned earlier that there are definitions of displacement structure that are different than the ones we use in this report. (See Chu and Kailath [1991] and Heinig and Rost [1984].) There are linear preserver questions analogous to the ones we studied here for the other definitions. We expect that the techniques that we have used here can be used to easily settle such analogous questions.

In the introduction of this report we noted that we are interested in the spectral properties of matrices that are Toeplitz or nearly Toeplitz. In particular, we are interested in sets having the following forms

$$
\operatorname{eigen}^{-1}(\lambda) \cap \operatorname{Toep}(m)
$$

or

$$
\text { eigen }^{-1}(\lambda) \cap \text { dis-inertia }^{-1}(p, n, z)
$$

where

$$
\begin{aligned}
\lambda & :=\left(\lambda_{1}, \ldots, \lambda_{m}\right) \in R^{m} \\
\operatorname{eigen}{ }^{-1}(\lambda) & :=\{A \in H \operatorname{erm}(m): \operatorname{eigen}(A)=\lambda\} \\
\operatorname{Toep}(m) & :=\left\{A \in C^{m \times m}: A \text { is } \operatorname{Toeplitz}\right\} \\
\text { dis-inertia }^{-1}(p, n, z) & :=\{A \in \operatorname{Herm}(m): \operatorname{dis-inertia}(A)=(p, n, z)\} .
\end{aligned}
$$

Now, by the spectral theorem, we have that

$$
\text { eigen }^{-1}(\lambda)=\left\{Q \operatorname{Diag}(\lambda) Q^{*}: Q \in U(m)\right\} .
$$

¿From this we see that linear spectra preserving operators

$$
\operatorname{Herm}(m) \longrightarrow \operatorname{Herm}(m): X \longrightarrow Q X Q^{*}
$$

for $Q \in U(m)$ can be used to move around this isospectral surface eigen ${ }^{-1}(\lambda)$. In more technical language, we see that eigen ${ }^{-1}(\lambda)$ is the orbit of $\operatorname{Diag}(\lambda)$ under the group action defined by

$$
U(m) \times \operatorname{Herm}(m) \longrightarrow \operatorname{Herm}(m):(Q, X) \longrightarrow Q X Q^{*} .
$$

We originally hoped that we could move around somewhat freely on the sets of the form eigen ${ }^{-1}(\lambda) \cap$ dis-inertia $^{-1}(p, n, z)$ by means of the linear preservers of such sets. This hope motivated our study of linear preservers. Unfortunately, our hope was too optimistic. Our results show that there are not enough such linear preservers. 


\section{REFERENCES}

[1] J. F. Adams, Vector fields on spheres, Ann. of Math., 75 (1962), pp. 603-632.

[2] J. F. Adams, P. D. Lax, And R. S. Phillips, On matrices whose real linear combinations are nonsingular, Proc. Amer. Math. Soc., 76 (1965), pp. 318-322.

[3] M. D. Atkinson, Primitive spaces of matrices of bounded rank II, J. Aust. Math. Soc. (Ser. A), 34 (1983), pp. 306-315.

[4] M. D. AtKInson and S. Lloyd, Large spaces of matrices of bounded rank, Quart. J. Math. Oxford, 31 (1980), pp. 253-262.

[5] - Primitive spaces of matrices of bounded rank, J. Aust. Math. Soc. (Ser. A), 30 (1981), pp. $473-482$.

[6] E. M. BARUCH AND R. Loewy, Linear preservers on spaces of Hermitian or real symmetric matrices, Preprint, (1991).

[7] L. B. BEASLEY, Linear transformations on matrices: the invaraiance of rank $k$ matrices, Linear Algebra and Its Applications, 3 (1970), pp. 407-427.

[8] - Linear transformations which preserve fixed rank, Linear Algebra and Its Applications, 40 (1981), pp. $183-187$.

[9] - Rank-k preservers and preservers of sets of ranks, Linear Algebra and Its Applications, 55 (1983), pp. 11-17.

[10] - Linear operators on matrices: the invariance of rank-k matrices, Linear Algebra and Its Applications, 107 (1988), pp. 161-167.

[11] P. BotTA, Linear maps preserving rank less than or equal to one, Linear and Multilinear Algebra, 20 (1987), pp. 197-201.

[12] M. T. CHU, The stability group of symmetric Toeplitz matrices, Preprint, (1992).

[13] J. Chun and T. KaIlath, Displacement structure for Hankel, Vandermonde and related (derived) matrices, Linear Algebra and Its Applications, 151 (1991), pp. 199-227.

[14] P. Comon, Displacement rank of pseudo-inverses, Preprint, (1992).

[15] D. Eisenbud and J. HarRis, Vector spaces of matrices of low rank, Advances in Math., 70 (1988), pp. 135-155.

[16] H. Flanders, On spaces of linear transformations with bounded rank, J. London Math. Soc., 37 (1962), pp. 10-16.

[17] A. Graham, Kronecker Products and Matrix Calculus: With Applications, Wiley, 1981.

[18] G. Heinig and K. Rost, Algebraic Methods for Toeplitz-like Matrices and Operators, Birkhauser, 1984.

[19] J. W. Helton and L. Rodman, Signature preserving linear maps of Hermitian matrices, Linear and Multilinear Algebra, 17 (1985), pp. 29-37.

[20] R. Horn and C. Johnson, Topics in Matrix Analysis, Cambridge University Press, 1991.

[21] R. HoRn, C. K. LI, AND N. K. Tsing, Linear operators preserving certain equivalence relations on matrices, SIAM J. Matrix Anal. Appl., 12 (1991), pp. 195-204.

[22] C. R. Johnson AND S. PIERCE, Linear maps on Hermitian matrices: the stabilizer of an inertia class, Canad. Math. Bull., 28 (1985),pp. 401-404.

[23] - Linear maps on Hermitian matrices: the stabilizer of an inertia class II, Linear and Multilinear Algebra, 19 (1986), pp. 21-31.

[24] T. KaIlath, S.-Y. Kung, and M. Morf, Displacement ranks of matrices, AMS Bull., 1 (1979), pp. $769-773$.

[25] - Displacement ranks of matrices and linear equations, J. Math. Anal. Appl., 68 (1979), pp. 395-407.

[26] T. J. LAFFEY AND R. LOEwY, Linear transformations which do not increase rank, Linear and Multilinear Algebra, 26 (1990), pp. 181-186.

[27] P. Lancaster and M. Tismenetsky, The Theory of Matrices: With Applications, Academic Press, 1985.

[28] M. H. LIM, Linear transformations of tensor spaces preserving decomposable tensors, Public. de l'Institut Math. (N.S.), 18 (1975), pp. 131-135.

[29] - Linear transformations on symmetric matrices, Linear and Multilinear Algebra, 7 (1979), pp. $47-57$

[30] R. LoEwy, Linear transformations which preserve or decrease rank, Linear Algebra and Its Applications, 12 (1989), pp. 151-161.

[31] - Linear maps which preserve a balanced nonsingular inertia class, Linear Algebra and Its Applications, 134 (1990), pp. 165-179.

[32] - Linear maps which preserve an inertia class, SIAM Journal on Matrix Analysis and Applications, 11 (1990), pp. 107-112.

[33] - Linear mappings which are rank-k nonincreasing, Preprint, (1991). (To appear in SIAM 
Journal Matrix Analysis and Applications).

[34] R. Loewy and S. Pierce, Linear preservers of balanced nonsingular inertia classes, Preprint, (1991).

[35] M. Marcus and B. Moyls, Transformations on tensor product spaces, Pacific J. Math., 9 (1959), pp. 1215-1221.

[36] R. Meshulam, On the maximal rank in a subspace of matrices, Quart. J. Math. Oxford, 36 (1985), pp. 225-229.

[37] - On two extremal matrix problems, Linear Algebra and Its Applications, 114-115 (1989), pp. 261-271.

[38] C. F. Moone, Characterization of transformations preserving rank two tensors of a tensor product space, Univ. of British Columbia, Vancouver B. C., Cananda, (1966).

[39] S. Pierce And L. Rodman, Linear preservers of the class of Hermitian matrices with balanced inertia, SIAM Journal on Matrix Analysis and Applications, 9 (1988), pp. 461-472.

[40] H. SCHNEIDER, Positive operators and an inertia theorem, Numer. Math., 7 (1965), pp. 11-17.

[41] J. Sylvester, On the dimension of spaces of linear transformations satisfying rank conditions, Linear Algebra and Its Applications, 78 (1986), pp. 1-10.

[42] R. Westwick, Transformations on tensor spaces, Pacific J. Math., 23 (1967), pp. 613-620.

[43] - Spaces of matrices of fixed rank, Linear and Multilinear Algebra, 20 (1987), pp. 171-174. 